\title{
MS43-P03 | STRUCTURES OF THE GROUP 13 PRENUCLEATION CLUSTERS
}

Gjerlevsen Nielsen, Ida (Center for Materials Crystallography, Aarhus University, Aarhus C, DNK); Sommer, Sanna (Center for Materials Crystallography, Aarhus University, Aarhus C, DNK); Brummerstedt Iversen, Bo (Aarhus University, Aarhus, DNK)

Commonly classical nucleation theory have been used to explain nucleation, but this is now being challenged as atomic scale techniques has been developed to study solutions showing larger clusters before nucleation. Thus, a new theory including prenucleation clusters (PNCs) with predictive value is needed. To achieve this, it is essential to settle the atomic structure of PNCs across numerous elements as well as chemical environment.

The group 13 metal oxides have been chosen as a starting system. Al, In and Ga form similar oxides and hydroxides such as $\mathrm{M}(\mathrm{OH})_{3}, \mathrm{MOOH}$ and $\mathrm{M}_{2} \mathrm{O}_{3}$ in solvothermal synthesis. The individual systems exhibit complex polymorphism, which can be controlled with different synthesis parameters such as solvent and temperature, however, the actual mechanisms are unknown.

The PNCs structures of the group 13 metal oxides have been determined by combining PDF and EXAFS analysis of the three metal nitrates in various solvents. Based on this, a synthesis pathway have been suggested which facilitate $y-\mathrm{Al}_{2} \mathrm{O}_{3}$ formation without intermediate $\mathrm{AlOOH}$, a material currently used in industry in large scale. 